\title{
2-D SENSOR POSITION PERTURBATION ANALYSIS: EQUIVALENCE TO AWGN ON ARRAY OUTPUTS
}

\author{
Volkan Cevher, James H. McClellan \\ Georgia Institute of Technology \\ Atlanta, GA 30332-0250 \\ cevher@ieee.org, \\ jim.mcclellan@ece.gatech.edu
}

\begin{abstract}
In this paper, the performance of a subspace beamformer, namely the multiple signal classification algorithm (MUSIC), is scrutinized in the presence of sensor position errors. Based on a perturbation model, a relationship between the array autocorrelation matrix and the source autocorrelation matrix is established. It is shown that under certain assumptions on the source signals, the Gaussian sensor perturbation errors can be modelled as additive white Gaussian noise (AWGN) for an array where sensor positions are known perfectly. This correspondence can be used to equate position errors to an equivalent signal-to-noise ratio (SNR) for AWGN in performance evaluation. Finally, Cramér-Rao bound for the position perturbations that can be computed using the Cramér-Rao bound relations for the additive Gaussian noise case at high SNR's.
\end{abstract}

\section{INTRODUCTION}

In array signal processing, subspace-based direction-of-arrival (DOA) algorithms such as the MUSIC algorithm are preferred due to their computational and resolution advantages. One of the main disadvantages of these algorithms, however, is their high sensitivity to various system errors such as gain and phase perturbations, mutual coupling effects, sensor positions, etc [1]-[4].

The assumption of perfect knowledge of the sensor positions is never met in practice. The available calibration algorithms tend to find the individual sensor or node positions within some uncertainty [5]. In this paper, we evaluate the performance of a subspace-based algorithm MUSIC in the presence of sensor position errors. The approach is statistical since the position perturbations are assumed to have

\footnotetext{
Prepared through collaborative participation in the Advanced Sensors Consortium sponsored by the U. S. Army Research Laboratory under the Collaborative Technology Alliance Program, Cooperative Agreement DAAD19-01-02-0008. The U. S. Government is authorized to reproduce and distribute reprints for Government purposes notwithstanding any copyright notation thereon.
}

a Gaussian pdf. Our formulation differs from the previous work since the position perturbations are taken to be timeinvariant.

The following assumptions are made in this paper. The number of signals $p$ is assumed to be known. Methods for estimating $p$ are readily available [7]-[8]. The source signal amplitudes and their autocorrelation structure are known or given (once an estimate of the DOA is available, the estimation of the source signal reduces to a least square fit [6].) Errors in the MUSIC DOA estimates can arise from a number of sources such as finite sample effects, an imprecisely known error covariance matrix, and a perturbed array manifold. Finite sample effects and the effects of an unknown error covariance matrix will be ignored in this paper to isolate the effects of the perturbation errors. These effects are considered in [6] and [1]-[3].

We will establish a relationship between the sensor perturbation error variance and the usual additive Gaussian noise error variance used in the common DOA estimation problems. Once this relation is established, we will show that the Cramér-Rao bound results derived for the common array model can be equivalently used to determine a lower bound for the DOA variance as a function of the perturbation errors. Simulation results will be provided to validate our arguments.

Organization of the paper is as follows: In section 2, the model is defined, then in section 3 we will establish a relationship between the Gaussian noise variance in an array with perfect sensor positions (sensor noise) and additive Gaussian noise; and the Gaussian noise variance in an array with perturbed sensor positions and no additive Gaussian noise (position noise). In section 4 , using the correspondence between the errors, we will find a Cramér-Rao bound for the perturbation errors without explicitly evaluating the bound. 


\section{BACKGROUND}

Consider an arbitrary array of $M$ sensors and $p$ radiating narrow-band sources observed by the array. Without loss of generality, assume that the sources and the array are coplanar and the sources are away from the sensors such that the far-field assumption is applicable on the wavefronts of the signals. It should be noted that this assumption also confines the DOA estimation problem to single parameter estimation for each source (e.g., azimuth angle only); however, the derivation is extendable to the multiple parameter case.

Let $\mathbf{x}_{i}$ denote the $i^{\text {th }}$ sensor position vector and $\mathbf{k}_{j}$ the wavevector of the $j^{\text {th }}$ signal:

$$
\begin{aligned}
& \mathbf{x}_{i}=\left[\begin{array}{ll}
x_{i, x}, & x_{i, y}
\end{array}\right]^{T} \quad i=1,2, \cdots, M \\
& \mathbf{k}_{j}=\left[\begin{array}{ll}
k_{j, x}, & k_{j, y}
\end{array}\right]^{T} \quad j=1,2, \cdots, p
\end{aligned}
$$

where the subscripts $(x, y)$ denote the horizontal and the vertical components of the vectors in the 2-D plane. Nominal sensor positions $\left(\mathbf{x}_{i}\right.$ 's) are assumed to be known; however, the array has no special configuration (e.g., circular or linear). The position matrix $\mathbf{X}$ is formed from the nominal sensor positions as follows:

$$
\mathbf{X}=\left[\begin{array}{llll}
\mathbf{x}_{1}, & \mathbf{x}_{2}, & \cdots, & \mathbf{x}_{M}
\end{array}\right]
$$

A steering vector associated with the array defines the complex array response for a source at DOA $\theta$, and has the familiar form for the $i^{\text {th }}$ source signal:

$$
\mathbf{a}\left(\theta_{i}\right)=\mathbf{a}\left(\mathbf{X}, \mathbf{k}_{i}\right)=\left[\begin{array}{c}
e^{-j \mathbf{k}_{\mathbf{i}}^{T} \mathbf{x}_{1}} \\
e^{-j \mathbf{k}_{\mathbf{i}}^{T} \mathbf{x}_{2}} \\
\vdots \\
e^{-j \mathbf{k}_{\mathbf{i}}^{T} \mathbf{x}_{M}}
\end{array}\right]
$$

where $i=1,2, \cdots, p$. Each steering vector a $\left(\theta_{i}\right)$ uniquely corresponds to a signal whose direction is the objective of the DOA estimation problem. The signal vector $\mathbf{s}(t)$ is a $p$ dimensional vector that consists of only narrow-band signals:

$$
\mathbf{s}(t)=\left[\begin{array}{llll}
\mathbf{s}_{1}(t), & \mathbf{s}_{2}(t), & \cdots, & \mathbf{s}_{p}(t)
\end{array}\right]^{T}
$$

where it is assumed that $p$ is known.

$$
\text { Let } \mathbf{w}_{i} \sim \mathcal{N}\left(\mathbf{0}, \sigma^{2}\left[\begin{array}{ll}
1 & 0 \\
0 & 1
\end{array}\right]\right) \text { for } i=1,2, \cdots, M \text { de- }
$$

note the displacements from the nominal sensor positions for each sensor $i$. The position perturbations are assumed to be i.i.d. Gaussian random variables and are independent of the signals or any additive noises that may occur at the sensor outputs. Different perturbations are chosen according to the above distribution when the DOA estimation experiment is repeated. Hence, during any DOA estimation process via some beamformer such as MUSIC, the perturbations are time-invariant in that the array output is generated using the same fixed perturbed position at all times $t=1,2, \cdots, N$.

The perturbed position matrix $\mathbf{W}$ is formed in a similar fashion to the position matrix $\mathbf{X}$ :

$$
\mathbf{W}=\left[\begin{array}{llll}
\mathbf{w}_{1}, & \mathbf{w}_{2}, & \cdots, & \mathbf{w}_{M}
\end{array}\right]
$$

Hence, the perturbed sensor positions, denoted by $\tilde{\mathbf{X}}$, are given by the simple addition $\tilde{\mathbf{X}}=\mathbf{X}+\mathbf{W}$. The $i^{\text {th }}$ steering vector associated with the perturbed sensor positions is $\tilde{\mathbf{a}}\left(\theta_{i}\right)=\mathbf{a}\left(\tilde{\mathbf{X}}, \mathbf{k}_{i}\right)$ and it is related to the nominal steering vector by a matrix multiplication:

$$
\begin{aligned}
\tilde{\mathbf{a}}\left(\theta_{i}\right) & =\boldsymbol{\Gamma}_{i} \mathbf{a}\left(\theta_{i}\right) \\
\boldsymbol{\Gamma}_{i} & \triangleq \Gamma\left(\mathbf{W}, \mathbf{k}_{i}\right) \quad i=1,2, \cdots, p
\end{aligned}
$$

From (3), it is easy to verify that the perturbation matrix $\boldsymbol{\Gamma}_{i}$ is the following:

$$
\boldsymbol{\Gamma}_{i}=\left[\begin{array}{cccc}
e^{-j \mathbf{k}_{\mathbf{i}}^{T} \mathbf{w}_{1}} & 0 & \cdots & 0 \\
0 & e^{-j \mathbf{k}_{\mathbf{i}}^{T} \mathbf{w}_{2}} & \ddots & \vdots \\
\vdots & \ddots & \ddots & 0 \\
0 & \cdots & 0 & e^{-j \mathbf{k}_{\mathbf{i}}^{T} \mathbf{w}_{M}}
\end{array}\right]
$$

For narrow-band signals, the array output vector $\mathbf{y}(t)$ can be written as follows:

$$
\mathbf{y}(t)=\mathbf{A}(\boldsymbol{\Theta}) \mathbf{s}(t)+\mathbf{n}(t) \quad t=1,2, \cdots, N
$$

In (8), $\mathbf{y}(t)$ is the noisy array output vector, $\mathbf{n}(t)$ is an additive noise (e.g., $\mathbf{n}(t) \sim \mathcal{N}\left(0, \sigma_{n}^{2}\right)$ ), and $\mathbf{A}(\boldsymbol{\Theta})$ consists of the steering vectors in the following manner:

$$
\mathbf{A}(\boldsymbol{\Theta})=\left[\begin{array}{llll}
\mathbf{a}\left(\theta_{1}\right), & \mathbf{a}\left(\theta_{2}\right), & \cdots, & \mathbf{a}\left(\theta_{p}\right)
\end{array}\right]
$$

where $\boldsymbol{\Theta}=\left[\begin{array}{llll}\theta_{1}, & \theta_{2}, & \cdots, & \theta_{p}\end{array}\right]^{T}$. It should be noted that the sensor positions must be perfectly known in order to define $\mathbf{A}(\boldsymbol{\Theta})$ for this model.

The signal and the array autocorrelation matrices are denoted by $\mathbf{R}_{\mathbf{s}}$ and $\mathbf{R}$, respectively; and are given by 


$$
\begin{aligned}
\mathbf{R}_{\mathbf{s}} & =E\left\{\mathbf{s}(t) \mathbf{s}^{H}(t)\right\} \\
\mathbf{R} & =E\left\{\mathbf{y}(t) \mathbf{y}^{H}(t)\right\}
\end{aligned}
$$

where $E\{\}$ is the expectation operator. In the derivation of the perturbed array autocorrelation matrix, no special structure is assumed for the signal autocorrelation matrix $\mathbf{R}_{\mathbf{s}}$. However, the results will simplify when the source signals are assumed to be uncorrelated (i.e., diagonal $\mathbf{R}_{\mathbf{s}}$ ).

\section{EFFECTS OF THE POSITION PERTURBATIONS ON THE ARRAY AUTOCORRELATION MATRIX}

The signal model for subspace estimation when the sensor positions are perturbed may be written similar to (8) as

$$
\tilde{\mathbf{y}}(t)=\tilde{\mathbf{A}}(\boldsymbol{\Theta}) \mathbf{s}(t)+\mathbf{n}(t) \quad t=1,2, \cdots, N
$$

where the steering matrix $\tilde{\mathbf{A}}(\boldsymbol{\Theta})$ is the following:

$$
\begin{aligned}
\tilde{\mathbf{A}}(\boldsymbol{\Theta}) & =\left[\begin{array}{llll}
\tilde{\mathbf{a}}\left(\theta_{1}\right), & \tilde{\mathbf{a}}\left(\theta_{2}\right), & \cdots, & \tilde{\mathbf{a}}\left(\theta_{p}\right)
\end{array}\right] \\
& =\left[\begin{array}{llll}
\boldsymbol{\Gamma}_{1} \mathbf{a}\left(\theta_{1}\right), & \boldsymbol{\Gamma}_{2} \mathbf{a}\left(\theta_{2}\right), & \cdots, & \boldsymbol{\Gamma}_{p} \mathbf{a}\left(\theta_{p}\right)
\end{array}\right]
\end{aligned}
$$

Our objective in finding a closed form expression for $\tilde{\mathbf{R}}$ is to elicit and evaluate the effects of the sensor position perturbations on the DOA estimates, given that only the nominal sensor positions are known. Let us reiterate the fact that the perturbations are assumed to be random variables at each trial of the DOA estimation and do not vary with time. Moreover, for simplicity, the additive noise vector $\mathbf{n}(t)$ in (11) is assumed to be zero to isolate the effects of the position perturbations. The general case is trivial once this derivation is done and hence is omitted here.

Using the definitions in (10) and (12), the autocorrelation matrix of the perturbed array can be written as

$$
\begin{aligned}
\tilde{\mathbf{R}} & =E\left\{\tilde{\mathbf{y}}(\mathbf{t}) \tilde{\mathbf{y}}^{\mathbf{H}}(\mathbf{t})\right\} \\
& =\sum_{i=1}^{p} \sum_{j=1}^{p} E\left\{\boldsymbol{\Gamma}_{i} \mathbf{a}\left(\theta_{i}\right) s_{i}(t) s_{j}^{*}(t) \mathbf{a}^{H}\left(\theta_{j}\right) \boldsymbol{\Gamma}_{j}^{H}\right\} \\
& =\sum_{i=1}^{p} \sum_{j=1}^{p} E\left\{\boldsymbol{\Gamma}_{i} \mathbf{a}\left(\theta_{i}\right) \mathbf{a}^{H}\left(\theta_{j}\right) \boldsymbol{\Gamma}_{j}^{H}\right\}\left[\mathbf{R}_{\mathbf{s}}\right]_{i, j}
\end{aligned}
$$

$[.]_{i, j}$ refers to the $i, j^{\text {th }}$ element of the matrix inside the brackets. In the last step of (13), we made use of the fact that the signals are independent of the perturbations. Since the signal term is a scalar, it can be taken out of the matrix multiplications, and hence by the independence assumption, the autocorrelation term appears.

Let us now explicitly evaluate the expectations for $[\tilde{\mathbf{R}}]_{m, n}$, using the definitions of steering vectors and the perturbation manifolds in (3) and (7):

$$
\begin{array}{r}
{[\tilde{\mathbf{R}}]_{m, n}=\sum_{i=1}^{p} \sum_{j=1}^{p} E\left\{e^{-j\left(\mathbf{k}_{\mathbf{i}}{ }^{T} \mathbf{w}_{m}-\mathbf{k}_{\mathbf{j}}{ }^{T} \mathbf{w}_{n}\right)}\right\}} \\
e^{-j\left(\mathbf{k}_{\mathbf{i}}{ }^{T} \mathbf{x}_{m}-\mathbf{k}_{\mathbf{j}}{ }^{T} \mathbf{x}_{n}\right)}\left[\mathbf{R}_{\mathbf{s}}\right]_{i, j}
\end{array}
$$

At this step, we will need the following result: $E\left\{e^{j \alpha u}\right\}=$ $e^{-\frac{\alpha^{2} \sigma^{2}}{2}}$ for a constant $\alpha$ and $u \sim \mathcal{N}\left(0, \sigma^{2}\right)$. Then, it follows that

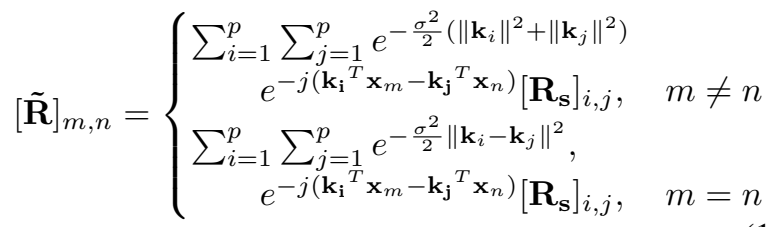

Equation (15) may be written in a compact way using the matrices as follows:

$$
\tilde{\mathbf{R}}=\mathbf{A}(\boldsymbol{\Theta}) \mathbf{D}(\boldsymbol{\Theta}) \mathbf{R}_{\mathbf{s}} \mathbf{D}^{\mathbf{H}}(\boldsymbol{\Theta}) \mathbf{A}^{\mathbf{H}}(\boldsymbol{\Theta})+\boldsymbol{\Sigma}(\boldsymbol{\Theta})
$$

where $\mathbf{D}(\theta)=\operatorname{diag}\left(\left[e^{-\frac{\sigma^{2}}{2}\left\|\mathbf{k}_{1}\right\|^{2}}, \quad \cdots, \quad e^{-\frac{\sigma^{2}}{2}\left\|\mathbf{k}_{p}\right\|^{2}}\right]\right)$ and

$$
\begin{array}{r}
{[\boldsymbol{\Sigma}(\theta)]_{m, n}=\delta_{m, n} \sum_{i=1}^{p} \sum_{j=1}^{p}\left[e^{-\frac{\sigma^{2}}{2}\left\|\mathbf{k}_{i}-\mathbf{k}_{j}\right\|^{2}}-e^{-\frac{\sigma^{2}}{2}\left(\left\|\mathbf{k}_{i}\right\|^{2}+\left\|\mathbf{k}_{j}\right\|^{2}\right)}\right]} \\
\times e^{-j\left(\mathbf{k}_{\mathbf{i}}^{T} \mathbf{x}_{m}-\mathbf{k}_{\mathbf{j}}{ }^{T} \mathbf{x}_{n}\right)}\left[\mathbf{R}_{\mathbf{s}}\right]_{i, j}
\end{array}
$$

It should be noted that when the position perturbation noise is chosen to be a uniform random variable, (15) has terms that depend on the individual wavevector components as opposed to its magnitude. Hence, the simplifications can not be made for uniform position perturbation noise.

Equation (17) simplifies if the signals are uncorrelated and have the same wavelength $\lambda$ :

$$
\boldsymbol{\Sigma}(\boldsymbol{\Theta})=\boldsymbol{\Sigma}=\sum_{i=1}^{p}\left|A_{i}\right|^{2}\left[1-e^{-\left(\frac{2 \pi \sigma}{\lambda}\right)^{2}}\right] \mathbf{I}
$$

where $A_{i}$ denotes the individual signal amplitudes and $\mathbf{I}$ is 
the $m \times m$ identity matrix. Under the same assumption, the array autocorrelation matrix assumes the form that we will concentrate on for the rest of this paper:

$$
\tilde{\mathbf{R}}=\frac{1}{\gamma}\left[\mathbf{A}(\boldsymbol{\Theta}) \mathbf{R}_{\mathbf{s}} \mathbf{A}^{H}(\boldsymbol{\Theta})+\sum_{i=1}^{p}\left|A_{i}\right|^{2}(\gamma-1) \mathbf{I}\right]
$$

where $\gamma=e^{\left(\frac{2 \pi \sigma}{\lambda}\right)^{2}}$.

Under the same assumptions leading to (19), it is also straight forward to derive the variance relation below:
Note that the variance of $[\mathbf{R}]_{m, n}$ can be shown to be [9]:

$$
\operatorname{Var}\left\{[\mathbf{R}]_{m, n}\right\}=\frac{1}{N} \sigma_{n}^{4}
$$

When (24) is compared to (20), one should notice that the variance of the diagonal elements in (24) is nonzero. Since the scaling factor $\gamma$ in (19) does not affect the noise and signal subspaces of the array autocorrelation matrix $\tilde{\mathbf{R}}$, it can be argued for MUSIC algorithm that the additive term on the right hand side of (19) can be, in fact, thought of as generated by an equivalent additive Gaussian noise with

$$
\operatorname{Var}\left\{[\tilde{\mathbf{R}}]_{m, n}\right\}=\left\{\begin{array}{cc}
\sum_{i=1}^{p} \sum_{j=1}^{p}\left[e^{-\sigma^{2}\left\|\mathbf{k}_{i}-\mathbf{k}_{j}\right\|^{2}}-e^{-\sigma^{2}\left(\left\|\mathbf{k}_{i}\right\|^{2}+\left\|\mathbf{k}_{j}\right\|^{2}\right)}\right] \\
\quad e^{-j\left(\mathbf{k}_{\mathbf{i}}-\mathbf{k}_{\mathbf{j}}\right)^{T}\left(\mathbf{x}_{m}-\mathbf{x}_{n}\right)}\left|A_{i}\right|^{2}\left|A_{j}\right|^{2} & m \neq n, \\
\mathbf{0} & m=n .
\end{array} \quad \sigma_{n}^{2}=2 N \sum_{i=1}^{p}\left|A_{i}\right|^{2}\left(e^{\left(\frac{2 \pi \sigma}{\lambda}\right)^{2}}-1\right)\right.
$$

In (20), it is interesting to note that $\tilde{\mathbf{R}}$ has zero variance on its diagonal due to the fact that sensor position perturbations lead to a constant bias on the time-delay information used in the DOA estimation for individual sensors when nominal sensor positions are used in the estimation process.

\section{APPLICATION: THE CRAMER-RAO BOUND}

The relationship between the array autocorrelation matrix and the source matrix in (19) suggests that the perturbation noise in the sensor positions may be considered as an additive white Gaussian noise term that could be added to (8). From (8), the usual derivation [6] leads to an array autocorrelation matrix that can be written as follows:

$$
\mathbf{R}=\mathbf{A}(\boldsymbol{\Theta}) \mathbf{R}_{\mathbf{s}} \mathbf{A}^{H}(\boldsymbol{\Theta})+\sigma_{n}^{2} \mathbf{I}
$$

where it is assumed that the noise vector is additive white gaussian noise (AWGN), i.e. $\mathbf{n}(\mathbf{t}) \sim \mathcal{N}\left(0, \sigma_{n}^{2}\right)$.

It is known that $\mathbf{R}$ is complex Wishart distributed with $N$ degrees of freedom and parameter matrix $\mathbf{P}=\frac{1}{N} \sigma_{n}^{2} \mathbf{I}$ (i.e., $\left.\mathbf{R} \sim \mathcal{W}_{M}^{C}(N, \mathbf{P})\right)[9]$ :

$$
f_{\mathcal{W}}(\mathbf{W})= \begin{cases}\frac{\operatorname{det}[\mathbf{W}]^{N-M}}{J(\mathbf{P})} e^{-\operatorname{trace}\left(\mathbf{P}^{-1} \mathbf{W}\right)} & \text { if } \mathbf{W}>0, \\ 0 & \text { otherwise }\end{cases}
$$

with

$$
J(\mathbf{P})=\pi^{M(M-1) / 2} \prod_{n=1}^{M} \Gamma(N-n+1)(\operatorname{det}[\mathbf{P}])^{N}
$$

where $f_{\mathcal{W}}(\mathbf{W})$ is the Wishart pdf for $\mathbf{W}$ and $\Gamma($.$) is the$ gamma function.
Equation (25) can be used to determine a Cramér-Rao bound for the DOA variance vs. the position perturbations. One important point to consider in this case is that since the perturbation noise is coherent for all snapshots and has $\sigma^{2}$ on both $x-y$ coordinate system, the signal-to-noise (SNR) ratio incorporates an additional $2 N$ factor.

Figure 1 displays the Cramér-Rao bound for the MUSIC algorithm as well as the performances of MUSIC for the additive Gaussian noise case and for the position-perturbation case.The Cramér-Rao bound is calculated using (5) in [11]. In Figure 1, the superimposed curve showing the performance of MUSIC under position perturbations closely follows the performance of MUSIC under additive Gaussian noise.

Note that in Fig. 1, the performance of MUSIC under AWGN differs from the performance of MUSIC under position perturbations at low equivalent SNR's. This result can be shown to hold also for conventional and minimumvariance beamformers. This can be attributable to the fact that the position perturbations result in a less severe effect on the distribution of array autocorrelation matrix than the AWGN. At high SNR's, the performance curves are very close due to the fact that the perturbations in sensor positions and the AWGN have the same first order effect on the array autocorrelation matrix.

\section{CONCLUSIONS}

In this paper, it is shown that sensor position perturbations create a highly signal dependent effect on the DOA estimation when multiple narrow-band signals are present. The distribution of the array autocorrelation matrix does not follow the Wishart distribution when large position noise is present. It is found that the effect of the AWGN on the autocorrelation matrix is much more severe than the effect of 


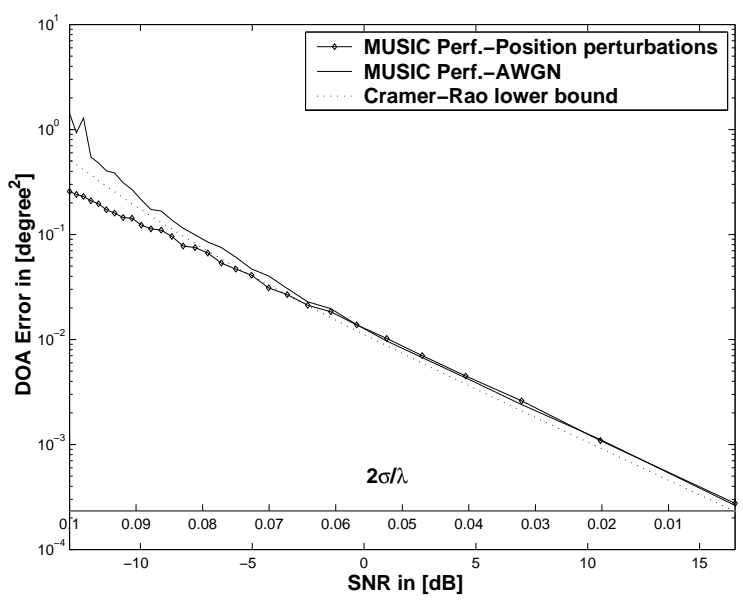

Fig. 1. 15-sensor uniform linear array with $\lambda / 2$ separation is used to verify the noise relation in (25). Sampling frequency of the data is $F_{s}=1000 \mathrm{~Hz}$, whereas the narrowband signals have a center frequency at $F=250 \mathrm{~Hz}$. Number of time samples is $N=100$ and the standard deviation of the sensor position perturbations is varied up to $10 \%$ of the inter-element spacing, i.e., $0.05 \lambda$.

the position perturbations. Direct derivation of the DOA estimation errors is not done in this paper and requires some further study. Hence, the Cramér-Rao bound under AWGN can be approximately used for the position perturbations at high SNR's (e.g., greater than 0dB).

\section{ACKNOWLEDGEMENTS}

The authors would like to thank Dr. Aaron Lanterman for his valuable comments on Wishart distributions.

\section{REFERENCES}

[1] A.L.Swindlehurst and T. Kailath, "A performance analysis of subspace-based methods in the presence of model errors, Part I: the MUSIC algorithm," IEEE Transactions on Signal Processing, vol. 40, no. 7, pp. 1758-1774, July 1992

[2] A.L.Swindlehurst and T. Kailath, "A performance analysis of subspace-based methods in the presence of model errors, Part II-Multidimensional algorithms," IEEE Transactions on Signal Processing, vol. 41, no. 9, pp. 2882-2890, September 1993

[3] B. Friedlander, "A sensitivity analysis of the MUSIC algorithm," IEEE Transactions on Acoustics, Speech, and Signal Processing, vol. 38, no. 10, pp.1740-1751, October 1990
[4] J.X. Zhu and H. Wang, "Effects of sensor position and pattern perturbations on CRLB for direction finding of multiple narrow-band sources," in Proc. 4th ASSP Workshop Spectral Estimation Modelling (Minneapolis, MN), pp.98-102, August 1988

[5] V. Cevher and J.H. McClellan, "Sensor array calibration via tracking with the extended Kalman filter," Acoustics, Speech, and Signal Processing, 2001. Proceedings. 2001 IEEE InternationalConference on, vol. 5, pp. 2817-2820, 2001

[6] P. Stoica and A. Nehorai, "MUSIC, maximum likelihood, and Cramer-Rao bound," IEEE Transactions on Acoustics, Speech, and Signal Processing, vol. 37, no. 5, pp.720-741, May 1989

[7] M. Wax and T. Kaliath, "Detection of signals by information theoretic criteria," IEEE Transactions on Acoustics, Speech, and Signal Processing, vol. ASSP33, pp.387-392, April 1985

[8] H. Wang and M. Kaveh, "On the performance of signal-subspace processing-Part I:Narrow-band systems," IEEE Transactions on Acoustics, Speech, and Signal Processing, vol. ASSP-34, pp. 1201-1209, October 1986

[9] D. Maiwald and D. Kraus, "Calculation of moments of complex Wishart and complex inverse Wishart distributed matrices," IEE Proc.-Radar, Sonar Navig., vol. 147, pp. 162-168, August 2000

[10] D.H.Johnson and D.E. Dudgeon, Array Signal Processing: Concepts and Techniques, chapter 4, Prentice Hall Signal Processing Series, 1993

[11] P. Stoica, E.G. Larsson, and A.B. Gershman, "The stochastic CRB for array processing: a textbook derivation," IEEE Signal Processing Letters, vol. 8, no. 5, pp. 148-150, May 2001 